\title{
Long-term outcomes of liver transplantation using grafts from donors with active and chronic hepatitis B virus infection
}

\author{
Sujin Gang ${ }^{1}$, YoungRok Choi ${ }^{1}$, Boram Lee ${ }^{2}$, Kyung Chul Yoon ${ }^{3}$, Suk Kyun Hong ${ }^{1}$, Hae Won Lee ${ }^{2}$, Namjoon Yi ${ }^{1}$, \\ Kwang-Woong Lee ${ }^{1}$, Kyung-Suk Suh ${ }^{1}$ \\ ${ }^{1}$ Department of Surgery, Seoul National University Hospital, Seoul, Korea
${ }^{2}$ Department of Surgery, Seoul National University Bundang Hospital, Seongnam, Korea
${ }^{3}$ Department of Surgery, Seoul Metropolitan Government-Seoul National University Boramae Medical Center, Seoul, Korea
}

Background: Liver grafts from donors with hepatitis B infection (HBV) have been expanded the donor pool under the hepatitis $B$ immunoglobulin (HBIG) and antiviral agents in the HBV endemic area. We report the long-term outcome of liver transplantation (LT) using grafts from donors with chronic HBV infection.

Methods: Among 2,260 LTs which were performed at Seoul National University (SNU) Hospital, SNU Bundang Hospital, and Seoul Metropolitan Government-SNU Boramae Hospital between January 2000 and April 2019, 26 cases (1.2\%) of LT using grafts from donors with $\mathrm{HBsAg}(+), \mathrm{HBeAb}(+)$ or $\mathrm{HBV} \mathrm{DNA}(+)$ were analyzed retrospectively.

Results: Sixteen deceased donor LT were performed with $\mathrm{HBsAg}(+)$ grafts. Ten living donor LT were performed with inactive HBV infected grafts: eight HBsAg (-), HBcAb (+), and HBV DNA (+) cases; and two cases with chronic HBV carrier with seroconversion HBsAg (-), HBsAg (+), and HBeAg (+). Recipients' mean age was 59.0 \pm 10.3 years old and model for end-stage liver disease (MELD) score was 19.9 \pm 8.4 . There were seven HBe-negative chronic hepatitis, 16 inactive HBV infections, two HBsAg seroconversion, and one HBV vaccinated state in recipients. Their mean follow-up period was $82.6 \pm 60.1$ months. All 10 recipients of living donor LT survived and were in good condition during follow-up. When compared with the patients who got transplantation with nonHBV infected grafts, the mortality rate was $30.8 \%(8 / 26)$ vs. $18.6 \%(387 / 2,076)$. But there was no difference in patient survival $(P=0.247$; log-rank test). Cause of death was infection and cancer recurrence. All survived patients were in inactive or resolved status for HBV infection. No graft failure was observed. HBV infection was thought to be effectively controlled by HBIG and antiviral medication.

Conclusions: With careful patient selection and effective post-LT therapy, liver grafts from HBV infected donors can be used safely and give an opportunity to increase the donor pool in HBV endemic areas.

Corresponding author: YoungRok Choi

E-mail: choiyoungrok@gmail.com

\section{(C) The Korean Society for Transplantation}

This is an Open Access article distributed under the terms of the Creative Commons Attribution Non-Commercial License (http://creativecommons.org/licenses/by-nc/4.0/) which permits unrestricted non-commercial use, distribution, and reproduction in any medium, provided the original work is properly cited. 